\title{
Comparison of serum and bronchoalveolar lavage fluid sialic acid levels between malignant and benign lung diseases Turgut Isitmangil ${ }^{* 1}$, Gulbu Isitmangil ${ }^{2}$, Yasemin Budak ${ }^{2}$, Recep Aydilek3 and Mehmet Kutlu Celenk3
}

Address: ${ }^{1}$ Department of Thoracic Surgery, GMMA Haydarpasa Training Hospital, Istanbul, Turkey, ${ }^{2}$ Department of Immunology, Yeditepe University School of Medicine, Istanbul, Turkey and 3Department of Respiratory Diseases, GMMA Haydarpasa Training Hospital, Istanbul, Turkey

E-mail: Turgut Isitmangil* - sitmangil@superonline.com; Gulbu Isitmangil - gulbu@ hotmail.com;

Yasemin Budak - yaseminbudak2000@yahoo.com; Recep Aydilek - recep@yeditepe.edu.tr; Mehmet Kutlu Celenk - mkcelenk@hotmail.com ${ }^{*}$ Corresponding author

\begin{abstract}
Background: It is known that tissue and serum sialic acid levels may be altered by malignant transformation. In this study, sialic acid levels were determined in bronchoalveolar lavage fluid (BAL) and serum in two groups of patients with lung cancer and non-malignant diseases of the lung.
\end{abstract}

Methods: Colorimetric methods were used for determination sialic acid in serum and in BAL samples. Flexible bronchoscopy was used to obtain the latter.

Results: Sialic acid levels in bronchoalveolar lavage fluid and serum did not show any statistically significant difference between subjects with malignant and the non-malignant lung diseases $(p>$ $0.05)$. Sialic acid levels were also unrelated to the stage and localization of the tumor $(p>0.05)$.

Conclusions: Sialic acid levels do not appear to be a good marker for discriminating malignant from non-malignant diseases of the lung.

\section{Background}

Sialic acid (N-acetylneuraminic acid; NANA) is composed of alkylated derivatives of the neuraminic acid, which are present in various mucoproteins and as the carbohydrate component of cell membrane glycolipids. The carbohydrate moiety characterizes the cohesive, adhesive and antigenic properties by its effect on cell-tocell contacts. These properties often change substantially after malignant transformation of a cell. In addition, development of malignancy could affect the serum and tissue sialic acid levels as well [1]. Sialic acid molecules can potentially inhibit intermolecular and intercellular interactions by virtue of their negative charge. However, they can also act as critical components of ligands recognized by a variety of animal, plant, and microbial proteins termed sialic acid binding lectins [2]. Sialic acid concentrations vary physiologically with age, but their levels may also be influenced by such conditions as inflammation, neoplastic tumors or inborn genetic disorders, which cause abnormal sialic acid metabolism [3].

In this study we intended to determine sialic acid levels in BAL fluid and serum in patients with lung cancer and non-cancer diseases of the lung and compare the results. 


\section{Methods}

A total of 40 patients were enrolled. Mean age was 50.6 (range: 19-75) with the male to female ratio being 20:1. Twenty-one patients (Group 1) had been diagnosed with lung cancer (Table 1), and the remaining 19 (Group 2) had various non-malignant lung diseases consisting of infections for the most part (Table 2). Informed consent was obtained from every patient participating the study.

We measured BAL fluid and serum sialic acid levels in each patient. BAL fluid was obtained in the same manner in all, utilizing flexible fiberoptic bronchoscopy. The upper respiratory tract was anesthetized using $2 \%$ lidocaine and atropine (0.75 $\mathrm{mg}$ IM) administered before the procedure. Two 50-mL aliquots of sterile saline, prewarmed to $37^{\circ} \mathrm{C}$, were infused into appropriate lung segments. Fluid was immediately recovered using gentle suction after introduction of each aliquot. BAL samples were obtained from the lobe containing the cancer in patients with lung cancer, from the segment(s) of greatest involvement in patients with pneumonia and lung tuberculosis, and from the right middle lobe in the setting of other diseases. The recovered fluid was passed through monolayer surgical gauze to eliminate mucus. Colorimetric method (Shimadzu UV-1601) was used for detection of sialic acid (Sigma, Ao812, Type IV-S). A 14-fold concentrate of lavage fluid was used in the measurement process; appropriate dilution factors were used in subsequent calculation of results.

\section{Results}

Mean sialic acid levels in BAL fluid of lung cancer patients were $10.31 \pm 6.53 \mathrm{mg} / \mathrm{dl}$ and $7.34 \pm 3.08 \mathrm{mg} / \mathrm{dl}$ in the non-cancer group. Mean serum sialic acid level in the former was $81.97 \pm 27.80 \mathrm{mg} / \mathrm{dl}$ and $72.05 \pm 23.18 \mathrm{mg} / \mathrm{dl}$ in the latter group. BAL fluid and serum sialic acid values of both patient groups are shown in Figure 1. Comparison of the values was made with the non-parametric Mann-Whitney test. BAL fluid sialic acid levels did not show a statistically significant difference between cancer and non-cancer groups $(p>0.05)$. Serum levels also failed to display any significant difference $(p>0.05)$ between the two groups. Moreover there was not a meaningful difference between sialic acid levels from the BAL fluids and sera of subjects at different stages of lung cancer ( $p>0.05)$. Lastly, neither did central or peripheral localization of the lung cancer confer any significant difference $(\mathrm{p}>0.05)$.

\section{Discussion}

Several studies have been performed about determination of different tumor markers in the serum and in BAL for the diagnosis of lung cancer and even for the type of tumor. Macchia et al (1987) have reported that the simultaneous determination of carcinoembryonic antigen

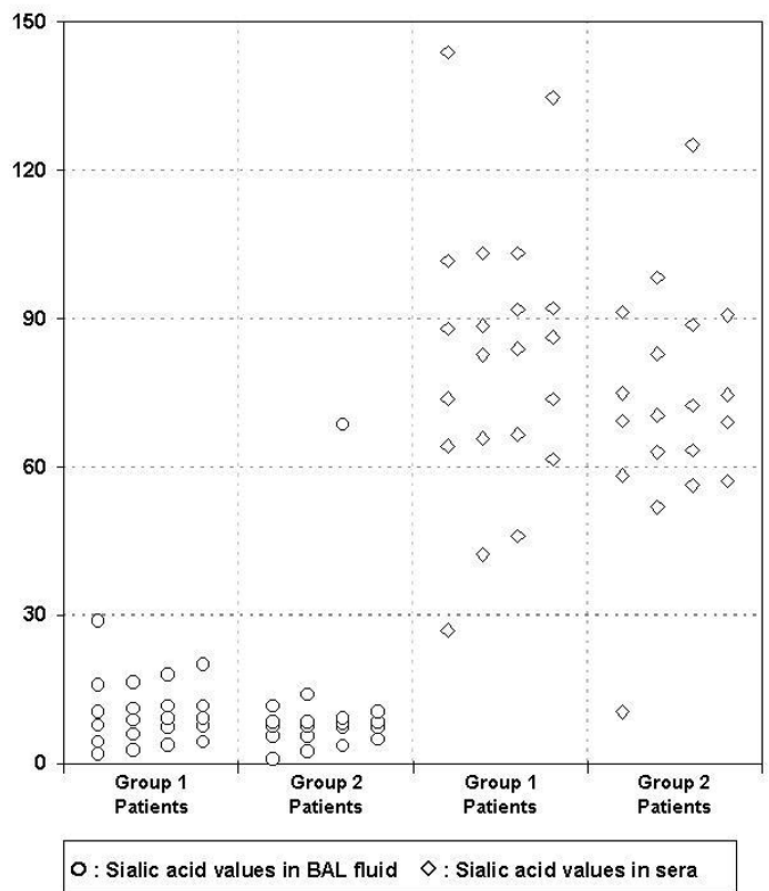

Figure I

Sialic acid values in BAL fluid and sera of each patient group.

(CEA) and CanAg CA-50 or neuron-specific enolase (NSE) in serum and in BAL is a useful aid in the diagnosis of lung malignancy [4]. To determine the usefulness of CEA in diagnosing lung cancer not visible endoscopically, de Diego et al (1991) compared CEA levels in BAL fluid and serum of patients with lung cancer, pneumonia, and healthy individuals [5]. They suggested that CEA determination in BAL fluid should not be used as the only diagnostic procedure. Niklinski et al (1992) declared that the simultaneous determination of CEA and squamous cell carcinoma antigen levels in serum and BAL in lung carcinoma patients might be of considerable importance in diagnosis [6]. To evaluate the diagnostic usefulness of simultaneous determinations of CEA, NSE, chorionic gonadotrophin (HCG), and carbohydrate antigenic determinant 19-9 (CA 19-9), Niklinski et al (1993) studied 48 patients with small cell lung carcinoma (SCLC) and 15 with nonmalignant lung disease [7]. The combination of CEA and NSE in BAL and NSE in serum taken together with results of bronchoscopy (histologic and cytologic) showed the highest discriminating power between malignant (SCLC) and nonmalignant lung disease [7].

Neuron-specific enolase is used in the staging and monitoring of responses to therapy and the detection of re- 
Table I: Sialic acid levels in BAL and sera samples of patients with malignant lung diseases (mg/dl).

\begin{tabular}{cccclll}
\hline Patient No. & Age & BAL Fluid & Serum & Diagnosis & Tumor Localization & Tumor Stage \\
\hline & & & & & & \\
I & 55 & 3.75 & 64.25 & Epidermoid carcinoma & Central & Stage IIB \\
2 & 50 & 1.87 & 88.60 & Epidermoid carcinoma & Central & Stage IIIA \\
3 & 64 & 5.97 & 73.69 & Epidermoid carcinoma & Central & Stage IIIA \\
4 & 68 & 11.09 & 143.96 & Epidermoid carcinoma & Peripheral & Stage IIIB \\
5 & 60 & 7.39 & 83.93 & Small cell carcinoma & Central & Stage IV \\
6 & 55 & 7.52 & 101.74 & Epidermoid carcinoma & Central & Stage IIB \\
7 & 72 & 7.78 & 134.70 & Small cell carcinoma & Central & Stage IV \\
8 & 59 & 8.8 & 46.07 & Adenocarcinoma & Peripheral & Stage IIIB \\
9 & 61 & 10.4 & 73.82 & Epidermoid carcinoma & Central & Stage IB \\
10 & 45 & 2.64 & 86.26 & Adenocarcinoma & Peripheral & Stage IIB \\
11 & 59 & 11.6 & 103.24 & Epidermoid carcinoma & Central & Stage IIIA \\
12 & 66 & 4.4 & 92.14 & Epidermoid carcinoma & Peripheral & Stage IIB \\
13 & 62 & 20 & 66.61 & Epidermoid carcinoma & Central & Stage IB \\
I4 & 48 & 16.4 & 103.24 & Epidermoid carcinoma & Central & Stage IIIA \\
I5 & 51 & 9.2 & 82.71 & Small cell carcinoma & Central & Stage IV \\
16 & 50 & 11.6 & 27 & Epidermoid carcinoma & Central & Stage IIIA \\
17 & 70 & 4.4 & 87.98 & Epidermoid carcinoma & Peripheral & Stage IIB \\
I & 48 & 17.9 & 91.86 & Adenocarcinoma & Peripheral & Stage IIIA \\
19 & 75 & 9.2 & 65.77 & Epidermoid carcinoma & Central & Stage IIIB \\
20 & 65 & 28.8 & 42.35 & Large cell carcinoma & Central & Stage IIIA \\
21 & 68 & 15.9 & 61.61 & Large cell Carcinoma & Central & Stage IIIB \\
& & & & & &
\end{tabular}

Table 2: Sialic acid levels in BAL and sera samples of patients with benign lung diseases $(\mathrm{mg} / \mathrm{dl})$.

\begin{tabular}{ccccl}
\hline $\begin{array}{c}\text { Patient } \\
\text { No. }\end{array}$ & Age & BAL Fluid & Serum & Diagnosis \\
& & & & \\
\hline 1 & 60 & 0.93 & 98.3 & Pneumonia \\
2 & 75 & 8.43 & 82.9 & Pneumonia \\
3 & 48 & 7.96 & 63.44 & Lung abscess \\
4 & 35 & 4.93 & 75 & Lung tuberculosis \\
5 & 40 & 5.6 & 63 & Pneumonia \\
6 & 24 & 9.2 & 51.90 & Lung tuberculosis \\
7 & 28 & 7.6 & 69.38 & Lung tuberculosis \\
8 & 49 & 7.2 & 91.31 & Pneumonia \\
9 & 30 & 11.6 & 69.11 & Sarcoidosis \\
10 & 34 & 2.4 & 56.34 & Lung tuberculosis \\
11 & 19 & 7.6 & 90.75 & Lung tuberculosis \\
12 & 60 & 8.4 & 72.44 & Lung tuberculosis \\
13 & 25 & 7.2 & 74.66 & Pneumonia \\
14 & 60 & 68.55 & 10.50 & COPD \\
15 & 43 & 8.4 & 57.17 & Lung tuberculosis \\
16 & 25 & 5.6 & 125.17 & Lung abscess \\
17 & 50 & 10.4 & 58.28 & Pneumonia \\
18 & 28 & 13.9 & 70.49 & Lung tuberculosis \\
19 & 43 & 3.6 & 88.81 & Pneumonia \\
& & & & \\
\hline
\end{tabular}

currences in lung cancer [8]. The diagnostic value of NSE has been under discussion. Prados et al. (1994) reported that NSE could be of aid in the early diagnosis of solitary pulmonary nodules and lung cancer [8]. Dowlati et al (1996) reported that high levels of NSE have recently been described in BAL fluid of patients with lung carcinoma [9]. BAL NSE is a better predictor of malignancy than serum NSE. BAL fluid measurements of NSE may have diagnostic value, especially if it is simultaneously measured in the serum [9].

Dowlati et al (1996) conducted a study to evaluate the usefulness of gastrin measurements in lung cancer and COPD patients and they found no difference between the gastrin levels in the BAL fluid or serum of the study groups [10]. There was no correlation with the stage in non-small cell lung carcinoma and no correlation was found between the gastrin levels in the serum and the BAL fluid. Therefore they concluded that there was no evidence of clinical usefulness for gastrin measurements in lung cancer [10].

Pina et al (2001) aimed to assess the diagnostic yield of the tumor markers carbohydrate antigen (CA125), CEA, NSE, squamous cell carcinoma antigen and specific tissue polypeptide antigen (TPS) in serum, BAL and biopsy cytosol in a group of patients with bronchogenic carcinoma [11]. This study suggested that determination of CEA 
in BAL was clinically the most useful marker in comparison with serum and cytosol determinations, although the latter may also be helpful in certain situations. Although there is no specific tumor marker for lung cancer, the combination of several can be used to monitor most patients with lung cancer [11].

Sialic acid levels have also been taken into account as a marker for lung cancer. Krolikowski et al (1976) showed that there is a significant difference in the levels of sialic acid in patients with lung cancer compared to normal controls. Hence they reported sialic acid to be of value as a biologic marker in lung cancer [12]. Seber et al (1989) also showed that sialic acid levels in BAL fluid were highly correlated with the diagnosis of bronchial cancer [13]. Kakari et al (1991) determined total sialic acid (TSA) and lipid-bound sialic acid (LSA) concentrations in comparison to CEA levels in 152 untreated patients with primary lung cancer and 107 benign pulmonary diseases. They concluded that although TSA and LSA were highly sensitive markers of lung cancer, their specificity was low in this setting [14]. Imecik et al (1992) measured sialic acid levels in the pleural effusions from patients with malignant and benign lung pathologies. Finding higher sialic acid concentrations in malignant pleural effusions than in those of benign origin, they concluded that the sialic acid had diagnostic value [15]. In another study by Patel et al (1995), carcinoembriyonic antigen (CEA) and phosphohexose isomerase (PHI) levels along with total sialic acid/total protein ratios (TSA/TP) in 192 untreated lung cancer patients and in 80 age- and sex-matched control cases were compared. PHI and TSA/TP were found to be as an important tumor marker in patients with lung cancer $[16]$.

We attempted in this study to more precisely define the potential role of sialic acid as a diagnostic marker in lung cancer by comparing its levels in malignant and benign conditions of the lung. However, we were not able to observe a clinically meaningful difference with respect to sialic acid concentrations between these two categories.

\section{Conclusions}

In this study, comparison of sialic acid levels in either BAL fluid or serum failed to show any statistically significant difference between patients with lung cancer and non-malignant, mostly infectious, pulmonary diseases. We therefore conclude that determination of sialic acid in the diagnostic evaluation of lung cancer needs more extensive study. Patients in remission might require a more cautious approach in this respect. We also believe that studies to clarify the exact role of sialic acid levels in the setting of inflammatory diseases will be useful as well.

\section{List of abbreviations}

$\mathrm{BAL}$ :Bronchoalveolar lavage

EA :Carcinoembriyonic antigen

COPD :Chronic obstructive pulmonary disease

HCG :Chorionic gonadotrophin

LSA :Lipid-bound sialic acid

NSE :Neuron-specific enolase

PHI :Phosphohexose isomerase

TPS :Tissue polypeptide antigen

TSA :Total sialic acid

\section{Competing interests}

None declared.

\section{Acknowledgement}

We are grateful to the staff of Hipokrat Laboratory in Istanbul for their help.

\section{References}

I. Christensen SE: Tumor markers. In Anderson SC, Cockayne S (eds). Clinical Chemistry. Concept and Applications. WB Saunders Company, Philadelphia, 1993322-336

2. Varki A: Sialic acids as ligands in recognition phenomena. FASEB J, 1997, I I:248-255

3. Fang-Kirscher SG: Comparison of sialic acids excretion in spot urines and 24-hour-urines of children and adults. Eur J Clin Chem Clin Biochem, 1997, 35:47-52

4. Macchia V, Mariano A, Cavalcanti M, Coppa A, Cecere C, Fraioli G, Elia S, Ferrante G: Tumor markers and lung cancer: correlation between serum and bronchial secretion levels of CEA, TPA, CanAg, NSE and ferritin. Int J Biol Markers, 1987, 2:151-156

5. de Diego A, Compte L, Sanchis J, Enguidanos MJ, Marco V: Usefulness of carcinoembryonic antigen determination in bronchoalveolar lavage fluid. A comparative study among patients with peripheral lung cancer, pneumonia, and healthy individuals. Chest, 1991, 100:1060-1063

6. Niklinski J, Chyczewska E, Furman M, Kowal E, Kozlowski M: Value of CEA and SCC-Ag in bronchoalveolar lavage (BAL) and serum of patients with lung carcinoma. Neoplasma, 1992, 39:283-5

7. Niklinski J, Chyczewska E, Furman M, Kowal E, Laudanski J, Chyczewski L: Usefulness of a multiple biomarker assay in bronchoalveolar lavage (BAL) and serum for the diagnosis of small cell lung cancer. Neoplasma, 1993, 40:305-308

8. Prados MC, Alvarez-Sala R, Blasco R, Chivato T, Garcia Satue JL, Garcia Rio FJ, Gomez de Terrero FJ, Villamor J: The clinical value of neuron-specific enolase as a tumor marker in bronchoalveolar lavage. Cancer, 1994, 74:1552-1555

9. Dowlati A, Bury T, Corhay JL, Weber T, Mendes P, Radermecker M: High neuron specific enolase levels in bronchoalveolar lavage fluid of patients with lung carcinoma: diagnostic value, relation to serum neuron specific enolase, and staging. Cancer, 1996, 77:2039-2043

10. Dowlati A, Bury T, Corhay JL, Weber T, Lamproye A, Mendes P, Radermecker $M$ : Gastrin levels in serum and bronchoalveolar lavage fluid of patients with lung cancer: comparison with patients with chronic obstructive pulmonary disease. Thorax I996, 5 I:I270-1272

II. Pina TC, Zapata IT, Hernandez FC, Lopez JB, Paricio PP, Hernandez $P M:$ Tumour markers in serum, bronchoalveolar lavage and 
biopsy cytosol in lung carcinoma: what environment lends the optimum diagnostic yield? Clin Chim Acta, 200I, 305:27-34

12. Krolowski FJ, Reuter K, Waales TP, Sieber SM, Adamson RH: Serum sialic acid levels in lung cancer patients. Pharmacol, 1976, | 4:47-5 |

13. Seber O, Aydilek R, Erbil K, Demirci N: Diagnostic value of lipidbound sialic acid levels in serum and bronchial lavage fluid in bronchial cancer. Asian Med J, 1989, 32:109-115

14. Kakari S, Stringou E, Toumbis M, Ferderigos AS, Poulaki E, Chondros $\mathrm{K}$, Dema A, Kotsovoulou V, Pavlidis N: Five tumor markers in lung cancer: significance of total and lipid-bound sialic acid. Anticancer Res, 1991, I I:2 107-21 I0

15. Imecik O, Ozer F: Diagnostic value of sialic acid in malignant pleural effusions. Chest, 1992, 102:1819-1822

16. Patel PS, Raval GN, Rawal RM, Patel GH, Balar DB: Comparison between serum levels of carcinoembryonic antigen, sialic acid and phosphohexose isomerase in lung cancer. Neoplasma, I995, 42:27I-274

Publish with BioMed Central and every scientist can read your work free of charge

"BioMedcentral will be the most significant development for disseminating the results of biomedical research in our lifetime." Paul Nurse, Director-General, Imperial Cancer Research Fund

Publish with BMC and your research papers will be:

- available free of charge to the entire biomedical community

- peer reviewed and published immediately upon acceptance

- cited in PubMed and archived on PubMed Central

- yours - you keep the copyright 